\title{
Three-Dimensional Dosimetry for Radiation Safety Estimates from Intrathecal Administration
}

\author{
Jacob Y. Hesterman ${ }^{1}$, Susan D. Kost ${ }^{2}$, Robert W. Holt ${ }^{1}$, Howard Dobson $^{1}$, Ajay Verma ${ }^{3}$, and P. David Mozley ${ }^{4}$ \\ ${ }^{1}$ inviCRO, Boston, Massachusetts; ${ }^{2}$ Cleveland Clinic, Cleveland, Ohio; ${ }^{3}$ Biogen, Cambridge, Massachusetts; and ${ }^{4}$ Weill Cornell \\ Graduate College of Medical Sciences, New York, New York
}

\begin{abstract}
Intrathecal administration is of growing interest for drug delivery, and its utility is being increasingly investigated through imaging. In this work, the 3-dimensional Voxel-Based Internal Dosimetry Application (VIDA) and 4D Extended Cardiac Torso Phantom (XCAT) were extended to provide radiation safety estimates specific to intrathecal administration. Methods: The 3-dimensional VIDA dosimetry application Monte Carlo simulation was run using a modified XCAT phantom with additional and edited cerebrospinal fluid (CSF) regions to produce voxel-level absorbed dose per unit cumulated activity maps for 9 selected source regions. Simulation validation was performed to compare absorbed dose estimates for common organs in a preexisting dosimetry tool (OLINDA/EXM). Dynamic planar imaging data were acquired in 6 healthy subjects using administered volumes of 5 or $15 \mathrm{~mL}$ ( $n=3$ each) of $185 \mathrm{MBq}$ of ${ }^{99 \mathrm{mTC}} \mathrm{Tc}$ diethylenetriaminepentaacetic acid. Absorbed dose was estimated for each subject using the intrathecal-specific dosimetry application. Results: Simulation results were within $6 \%$ of OLINDA estimates for common organs. Absorbed dose estimates were highest (0.3-0.8 mGy/MBq) in the lumbar CSF space. A whole-body effective dose estimate of $0.003 \mathrm{mSv} / \mathrm{MBq}$ was observed. An administered volume dependency was observed with a $15-\mathrm{mL}$ volume, resulting in lower absorbed dose estimates for several intrathecal and nonintrathecal regions. Conclusion: The intrathecal-specific VIDA implementation enables tailored dosimetry estimation for regions most relevant in intrathecal administration. Absorbed doses are highly localized to CSF and spinal regions and should be taken into consideration when designing intrathecal imaging studies. A potentially interesting relationship was observed between absorbed dose and administered volume, which merits further investigation.
\end{abstract}

Key Words: intrathecal administration; dosimetry; radiation safety

J Nucl Med 2017; 58:1672-1678

DOI: 10.2967/jnumed.117.190611

\section{I}

ntrathecal administration has emerged as a potentially attractive method to circumvent the blood-brain barrier for drug delivery in neurooncology and the treatment of some neurodegenerative disorders with large molecules (1). However, pharmacodynamics within the leptomeningeal space, including fluid flow of the cerebrospinal fluid (CSF) and associated clearance pathways, are complex and not fully understood. Imaging has begun to play a critical role in

Received Jan. 31, 2017; revision accepted Mar. 13, 2017.

For correspondence or reprints contact: Jacob Hesterman, inviCRO, LLC, 27 Drydock Ave., Boston, MA 02210.

E-mail: hesterman@invicro.com

Published online Mar. 23, 2017.

COPYRIGHT (C 2017 by the Society of Nuclear Medicine and Molecular Imaging. increasing the understanding of this system, including investigation of pulsatile CSF flow and its relationship to blood flow (2,3), respiration (4), and underlying pathway system $(5,6)$.

Although many of these studies have used MRI, radionuclide imaging methods, including PET and SPECT, are also valuable longitudinal imaging options because of their sensitivity and the useful half-life of several commonly used radionuclides. PET, SPECT, and planar imaging have recently been used, particularly in preclinical studies, to improve the understanding of CSF dynamics and drug delivery (7). This work has increased interest in the use of nuclear methods for clinical imaging applications but has also raised questions about potential risks from radiation dose (8).

Radiation dosimetry estimates are an important safety consideration when imaging with ionizing radiation. Dosimetry methods are well established, with tools such as OLINDA/EXM (9) routinely used in the development and evaluation of new and existing radiotracers $(10,11)$. Modern computing methods have begun to shift the dosimetry estimation landscape with an increased emphasis being placed on personalized dosimetry estimation, particularly for radiation therapy. Voxel-based tools have emerged that enable more granular estimation of radiation dose, including image-based approaches and associated tools such as SIMDOSE, LundADose, 3D-ID, 3D-RD, RMDP, and voxel-based internal dosimetry application (VIDA) (12-21).

Most commonly used dosimetry methods have been implicitly developed to accommodate rapid biodistribution in the systemic circulation, particularly from intravenous injections, because practicality has restricted the inclusion of source and target regions to the most relevant organs affected via that administration route. This report describes the implementation of VIDA to estimate the radiation safety profiles of radiopharmaceuticals that are administered intrathecally. The XCAT male and female phantoms were modified to include CSF compartments and used for 3-dimesional (3D) anatomic modeling for the 3D absorbed dose calculations. Validation of the absorbed dose results was performed using OLINDA/EXM. The intrathecal-extended model was also used to assess absorbed dose to subjects receiving ${ }^{99 \mathrm{~m}} \mathrm{Tc}$-diethylenetriaminepentaacetic acid ( ${ }^{99 \mathrm{~m}} \mathrm{Tc}$-DTPA) in varying administered volumes. Results from the simulation experiments and application to clinical data are presented. The discussion focuses primarily on the interpretation of the organ-specific absorbed dose values, (whole-body) effective dose, and their biologic implications.

\section{MATERIALS AND METHODS}

\section{Data Acquisition}

Human Participants. All procedures were approved by the Institutional Review Board of Weill Cornell Medical College. All participants gave informed consent, which was documented in writing. 
Selection criteria included being subjectively healthy and the absence of any major medical problems by history, on-study physical examination, a set of clinical laboratory studies, and repeated urine drug screens. MR images were used to exclude participants who had any relative contraindication to lumbar puncture.

Radiopharmaceutical. ${ }^{99 \mathrm{~m}} \mathrm{Tc}-\mathrm{DTPA}$ was diluted in artificial CSF under a study-specific Investigational New Drug (IND) application approved by the Food and Drug Administration (FDA) exclusively for use in this study. Current good manufacturing practice was used. All activity preparations were shown to pass quality control specifications for radiochemical purity. All activity preparations were prospectively shown to pass tests of pyrogenicity before administration. Sterility tests were performed post hoc.

\section{Imaging and Activity Quantification}

Healthy volunteers were intrathecally administered an activity of $185 \mathrm{MBq}$ of ${ }^{99 \mathrm{~m}} \mathrm{Tc}$-DTPA in artificial CSF volumes of 5 or $15 \mathrm{~mL}$ ( $n=3$ per cohort). The injection site was between L2/L3 or L3/L4 for all subjects. Starting at about $30 \mathrm{~min}$ after administration, no fewer than 12 serial whole-body planar images were acquired during the first $6 \mathrm{~h}$ after administration, with a final planar acquisition at approximately $22 \mathrm{~h}$ after injection. Data were acquired using a continuous-bed-motion acquisition on a 2-head Philips Forte camera, with a $20 \%$ energy window width centered at $140 \mathrm{keV}, 1,024 \times 512$ matrix size, and 2.00-mm pixel size. Whole-body scans were acquired with either a 6 -min or 12-min scan duration, except for the last scan, which had a 24-min scan duration. Selected frames from an example acquisition are shown in Figure 1. A transmission image was acquired for attenuation correction. Geometric means were calculated from conjugate anterior and posterior images. The dynamic, planar images were coregistered to the transmission scans and corrected for acquisition duration, decay, and attenuation. The transmission scan was also used as an anatomic reference image to place 18 regions of interest, including CSF (lumbar, lower thoracic, upper thoracic, cervical, cisterna magna), brain (substantia nigra, midbrain, motor cortex, frontal, hippocampus, pontomedullary junction, and remainder), left and right kidneys, bladder, waste bottle, background, and standards. A background region placed

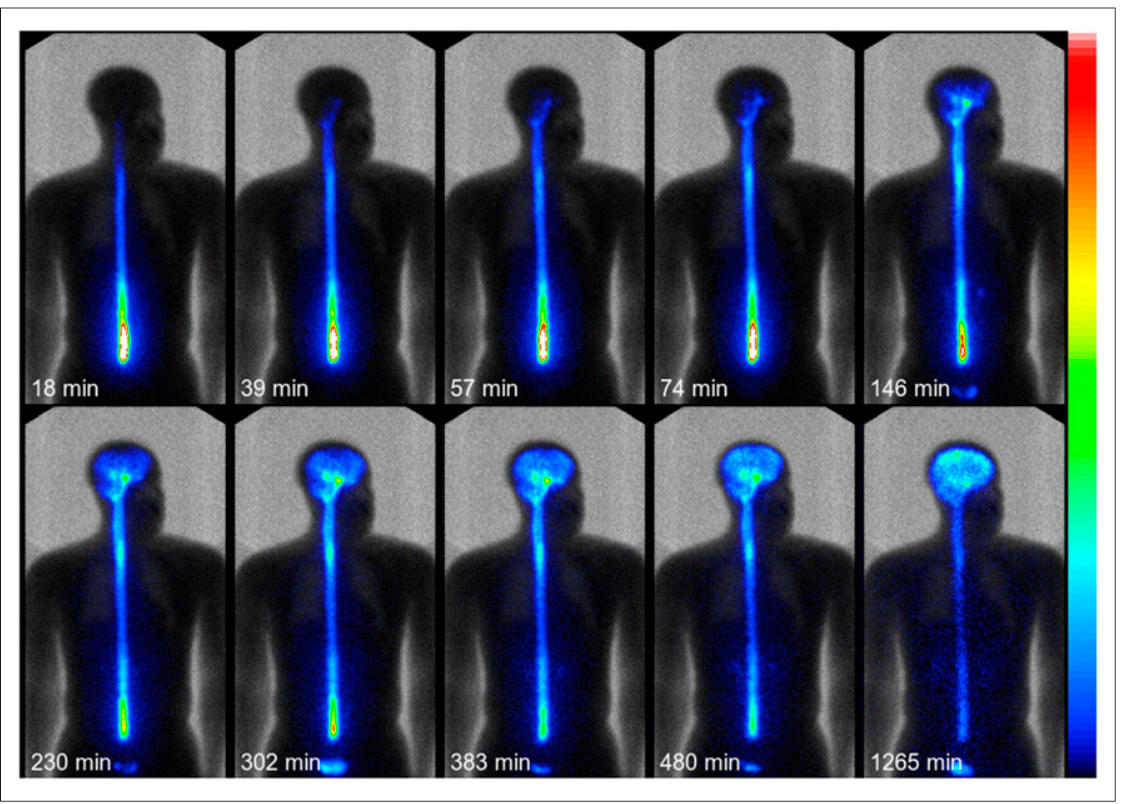

FIGURE 1. Example time-activity curve from select subject (5- $\mathrm{mL}$ dose volume), including time after administration. Gray scale represents transmission image, and color represents planar imaging data (black $\rightarrow 0$, white $\rightarrow 300 \mathrm{AU}$ ). superior to the left shoulder of the participants was used to subtract background noise. The activity map for each time point was normalized by the total integrated signal at the earliest time point and multiplied by 100 to create a percentage injected activity map. The earliest time point image available was chosen under the assumption that, because no waste was eliminated by any participant, the integrated activity was representative of the entire injected activity.

\section{XCAT Phantom}

The adult male and female non-uniform rational basis spline-based XCAT phantoms (22) were used as anatomic models for calculating 3D absorbed doses. Organ maps were created by rendering the male and female phantom in voxel format with resolution of $3.0 \times 3.0 \times 3.0 \mathrm{~mm}$. The rendering process also creates bone marrow cavities inside the phantom skeleton by eroding the outer bone layer by a cortical bone thickness that varies for each bone. The voxels within the marrow cavities were randomly populated as either red or yellow marrow in proportion with bone-specific marrow cellularity for a 40-y-old adult (23).

The phantoms include CSF compartments-the brain ventricles, cervical, upper thoracic (T1-T6), lower thoracic (T7-T12), and lumbar vertebrae canals, which were edited on the basis of analysis of the 6 subjects enrolled in the study. Spinal CSF regions were segmented from each subject's T2-weighted MR datasets, using heuristic threshold optimization to account for bias-field artifacts. The segmentation was manually edited to account for any remaining artifacts, split into anatomic subregions of interest, and used to estimate spinal CSF volume in each. The CSF region encompasses both the fluid and the spinal cord as limitations in both phantom and data resolution, particularly for the nuclear medicine data, limit the utility of separating these 2 regions. Spinal CSF regions in the XCAT phantom were modified to reflect the average volumes from the MR-based segmentation. Additionally, vertebral bone regions were split into subregions corresponding to the CSF regions. Examples of spinal bone and CSF regions are shown in Figure 2.

\section{Monte Carlo Simulation}

VIDA (20), a GEANT4-based Monte Carlo simulation code for targeted radionuclide therapy, was used to generate $3 \mathrm{D}$ absorbed dose maps. VIDA performs a voxel-by-voxel absorbed dose calculation for any radionuclide through direct sampling of its decay scheme. Material definitions used in the simulation are provided in Table 1 and were derived from data tabulated in International Commission on Radiation Units and Measurements publication 46 appendix A (24).

Nine anatomic regions were defined as sources of activity, including brain, brain ventricles, cervical, upper thoracic (T1-T6), lower thoracic (T7-T12), and lumbar CSF spaces, left and right kidneys, and bladder contents. For each source volume, 10 million decay events were generated assuming a uniform distribution of ${ }^{99 \mathrm{~m}} \mathrm{Tc}$ within the source. For each source region, a 3D absorbed doserate map was obtained by converting the $3 \mathrm{D}$ deposited energy map generated by VIDA using material densities in Table 1.

\section{VIDA Simulation Validation}

Validations of simulation results of ${ }^{99 \mathrm{~m}} \mathrm{Tc}$ by VIDA were performed using 2 independent techniques. Self-dose factors (absorbed dose per unit cumulated activity) in unit-density spheres ranging in mass from 10 to $1,000 \mathrm{~g}$ 


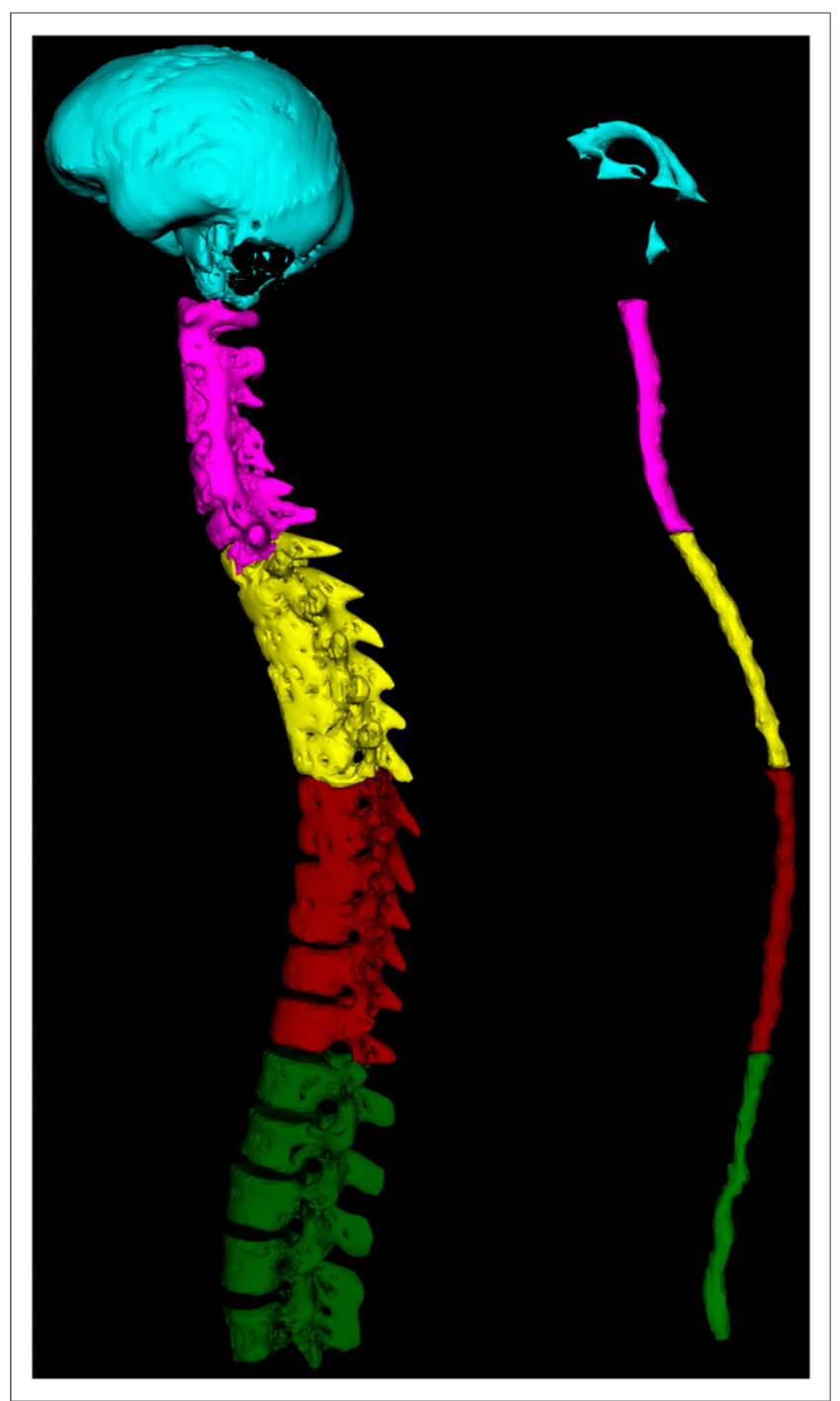

FIGURE 2. Brain and spine bone/CSF regions, including brain (cyan), cervical (fuchsia), upper thoracic (yellow), lower thoracic (maroon), and lumbar (green) regions.

were calculated using the energy deposited within the sphere from 1 million uniformly distributed decay events. The spheres comprised unit-density soft tissue $(25,26)$ surrounded by a semiinfinite volume of water. The self-dose factors were compared with the unit-density sphere model in OLINDA/EXM (11).

Self-dose factors for 2 sources, kidneys and brain, were calculated from the 3D dose maps for the male and female XCAT phantoms. These self-dose factors were compared with values from OLINDA/ EXM. The phantom organ masses in OLINDA/EXM were modified to be equal to those in the voxelized XCAT phantoms before the selfdose factors were calculated.

\section{Dosimetry}

Percentage injected activity estimates from each region were computed for each available time point. Trapezoidal integration was used for computation of the mean residence time from the imaging data, including assumption of zero activity at time zero for all regions outside of the injection site. Only physical decay was considered for computation of mean residence time from the last time point at approximately $22 \mathrm{~h}$ after injection onward. Calculated region mean residence times were multiplied by their corresponding absorbed dose-rate maps to generate voxel-level absorbed dose per unit injected activity maps for each source region. These maps were summed and multiplied by the injected activity to produce a scanand injection-specific voxel-level absorbed dose map. Mean and maximum absorbed dose values were estimated at the region level for each subject. The International Commission on Radiological Protection (ICRP) publication 103 weighting factors were applied for effective dose computation (23).

\section{RESULTS}

\section{Data Acquisition}

The final sample consisted of 6 men with a mean age of $45.3 \pm$ 4.5 y (minimum, 36.6; maximum, 49.7). All participants tolerated the procedures reasonably well; over the first $24 \mathrm{~h}$ after lumbar puncture, there were no subjective complaints of pain, and repeated physical examinations revealed no bleeding, bruising, or swelling for any subject. Lateral planar images at the 60-minafter-injection mark as well as SPECT images at the 8- and 22-h marks showed that there were no extravasations of the activity outside the intrathecal spaces.

\section{VIDA Simulation Validation}

Self-dose factors to spheres with uniform activity of ${ }^{99 \mathrm{~m}} \mathrm{Tc}$ between VIDA and OLINDA/EXM were compared for 10-, 20-, 100-, and 400-g spheres. Percentage differences between the 2 tools ranged from $-0.5 \%$ for a $10 \mathrm{~g}$ sphere to $2.3 \%$ for a 400 -g sphere. The comparison of self-dose factors calculated in the voxelized male and female XCAT phantom using VIDA to those from the stylized Snyder-Fisher phantom used in OLINDA/EXM are shown in Table 2. Deviations in the selfdose factors for kidney and brain ranged between $0.7 \%$ and $5.7 \%$.

\section{Clinical Data}

Example absorbed dose-rate maps for lumbar CSF and brain regions are shown in Figure 3. Mean residence times for the 5-mL group and 15-mL group were estimated as $6.49 \pm 0.75$ and $6.07 \pm$ $0.24 \mathrm{~h}$, respectively. An example composite voxel-level absorbed dose map is shown in Figure 4, and group-level average absorbed dose estimates are shown for several regions in Table 3. Lung, liver, stomach, and bladder regions are estimated as target regions and were not used as source regions in this study because of the low amounts of radioactivity seen in those regions. Effective dose estimates, calculated using ICRP 103 weighting factors, are also shown in Table 3.

\section{DISCUSSION}

The extension of VIDA to support data from intrathecal administration and resulting data from simulation and clinical application are discussed, including potential biologic implications and possible refinements or additional extension of the application.

\section{Simulation Findings}

VIDA was previously validated for common radionuclides used in targeted radionuclide therapy, including ${ }^{131} \mathrm{I},{ }^{90} \mathrm{Y}$, and ${ }^{177} \mathrm{Lu}(20)$. The same methods were used to verify the 3D absorbed doses for ${ }^{99 \mathrm{~m}} \mathrm{Tc}$ calculated in this study. The agreement in self-dose factors for uniform activity spheres was within $3 \%$ compared with the sphere model in OLINDA/EXM. Two organs 
TABLE 1

Summary of Materials Used in Monte Carlo Simulation Model

\begin{tabular}{|c|c|c|c|c|c|c|c|c|c|c|c|}
\hline \multirow[b]{2}{*}{ Material } & \multirow[b]{2}{*}{ Density $\left(\mathrm{g} / \mathrm{cm}^{3}\right)$} & \multicolumn{10}{|c|}{ Chemical composition ( $\%$ by mass) } \\
\hline & & $\mathrm{H}$ & $\mathrm{C}$ & $\mathrm{N}$ & $\mathrm{O}$ & $\mathrm{Na}$ & $\mathrm{P}$ & $S$ & $\mathrm{Cl}$ & K & Other \\
\hline Air & $1.21 \times 10^{-3}$ & & 0.01 & 75.5 & 23.2 & & & & & & $1.3(\mathrm{Ar})$ \\
\hline Soft tissue & 1.03 & 10.5 & 25.6 & 2.7 & 60.2 & 0.1 & 0.2 & 0.3 & 0.2 & 0.2 & \\
\hline Lung tissue & 0.26 & 10.3 & 10.5 & 3.1 & 74.9 & 0.2 & 0.2 & 0.3 & 0.3 & 0.2 & \\
\hline Mammary gland & 1.06 & 10.2 & 15.8 & 3.7 & 69.8 & 0.1 & 0.1 & 0.2 & 0.1 & 0.1 & \\
\hline Adipose tissue & 0.95 & 11.4 & 59.8 & 0.7 & 27.8 & 0.1 & & 0.1 & 0.1 & & \\
\hline Water (CSF) & 1.00 & 11.1 & & & 88.9 & & & & & & \\
\hline Cortical bone (40 y) & 1.92 & 3.4 & 15.5 & 4.2 & 43.5 & 0.1 & 10.3 & 0.3 & & & $0.2(\mathrm{Mg}), 22.5(\mathrm{Ca})$ \\
\hline Cartilage & 1.10 & 9.6 & 9.9 & 2.2 & 74.4 & 0.5 & 2.2 & 0.9 & 0.3 & & \\
\hline Red marrow & 1.03 & 10.5 & 41.4 & 3.4 & 43.9 & & 0.1 & 0.2 & 0.2 & 0.2 & $0.1(\mathrm{Fe})$ \\
\hline Yellow marrow & 0.98 & 11.5 & 64.4 & 0.7 & 23.1 & 0.1 & & 0.1 & 0.1 & & \\
\hline
\end{tabular}

available in the stylized phantom series of Cristy and Eckerman (27) implemented in OLINDA/EXM were used as source regions in this study, the kidneys and brain. The self-doses to the kidneys and brain agree within $6 \%$ to self-doses from OLINDA/ EXM after the reference phantom organ masses were adjusted. The difference between the average absorbed dose deposited to the source organ by VIDA and reference data from OLINDA/ EXM is due to several factors. The stylized phantoms used in OLINDA/EXM have organs defined by geometric shapes compared with the more anatomically accurate XCAT phantom. The tissue compositions and densities in VIDA may have slight differences compared with those used to generate the specific absorbed fractions for monoenergetic photons and electrons that are weighted by OLINDA/EXM to generate the absorbed dose specific to the decay spectrum for each radionuclide of interest. Given these differences, the agreement between VIDA and OLINDA/EXM for self-dose factors for the kidneys and brain in both the male and the female XCAT is excellent.

\section{Clinical Data}

Qualitative review of the image data as well as the relatively high residence times ( $>6 \mathrm{~h}$ for both groups) suggest a slow systemic clearance of ${ }^{99 \mathrm{~m}}$ Tc-DTPA. Generally, lower absorbed dose values were observed in the $15-\mathrm{mL}$ administered volume group than the 5-mL group. These differences were statistically significant at an $\alpha$-threshold of 0.05 when comparing 5 - and 15-mL administered volume groups for several regions, including lumbar and lower thoracic CSF, lungs, liver, kidneys, stomach, and heart. Additional subjects per group and use of a crossover study design would be beneficial to strengthen these findings and improve understanding around why the lower values are observed in the $15-\mathrm{mL}$ group. This finding is consistent with administered volume effects observed in preclinical studies of rats and nonhuman primates in that larger administered volumes tend to fill the CSF space and proportionally reach the brain and clearance routes more rapidly $(7,28)$.

The lumbar CSF region experiences the highest exposure, with an absorbed dose per unit injected activity of approximately $0.8 \pm 0.2 \mathrm{mGy} / \mathrm{MBq}$ for a $5-\mathrm{mL}$ administered volume, with radiation dose decreasing along the spinal cord up to the brain. In the case of the 5-mL administration, the ratio of absorbed dose between lumbar and cervical CSF is approximately 5.5 and lumbar to brain tissue is about 23. A more uniform distribution of absorbed dose is observed with the $15-\mathrm{mL}$ dose volume with lumbar-to-cervical and lumbar-to-brain tissue ratios of about 2.1 and 7.4, respectively. Additionally, the brain CSF, comprising largely the cisterns, and brain parenchyma doses are approximately 1.4 times higher in the $15-\mathrm{mL}$ administered volume group than in the 5-mL group.

Regions outside the CSF and brain receive relatively low absorbed dose, with the kidneys receiving the highest absorbed dose. On average, $91 \%$ of the total kidney-absorbed dose results from a combination of self-dose due to DTPA clearance and cross-radiation from the lumbar region. For the $5-\mathrm{mL}$ group, $34 \%$ of the total kidney-absorbed dose is due to crossradiation from the lumbar region compared with $22 \%$ in the

TABLE 2

Self-Dose Factor Comparison Between VIDA DF and OLINDA

\begin{tabular}{llcrr}
\hline Phantom & Organ & VIDA DF (mGy/MBq-s) & OLINDA (mGy/MBq-s) & $\%$ difference \\
\hline Male & Kidneys & $1.186 \mathrm{E}-05$ & $1.194 \mathrm{E}-05$ & $0.7 \%$ \\
& Brain & $4.925 \mathrm{E}-06$ & $4.556 \mathrm{E}-06$ & $5.7 \%$ \\
Female & Kidneys & $1.337 \mathrm{E}-05$ & $1.358 \mathrm{E}-05$ & $1.6 \%$ \\
& Brain & $4.627 \mathrm{E}-06$ & $4.861 \mathrm{E}-06$ & $4.8 \%$ \\
\hline
\end{tabular}




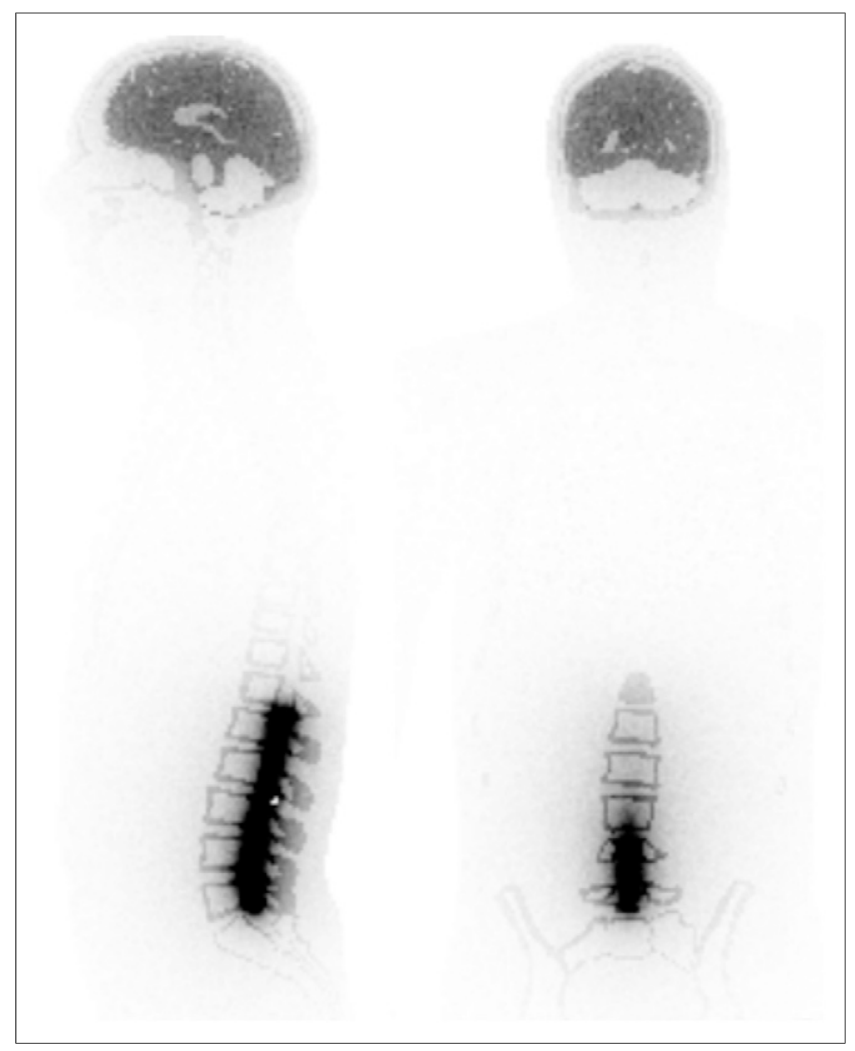

FIGURE 3. Example absorbed dose per unit cumulated activity for brain and lumbar regions (maximum, $8 \mathrm{e}-6 \mathrm{mGy} / \mathrm{MBq}$ s).

15-mL group. Additionally, the 15-mL administered volume group exhibits lower total absorbed dose in the kidneys than the $5-\mathrm{mL}$ group. The difference is due to both lower kidney self-dose $(\sim 15 \%$ lower in 15 than $5 \mathrm{~mL})$ and, particularly, a smaller contribution from the lumbar region $(\sim 200 \%$ lower in 15 than $5 \mathrm{~mL}$ ). Because of the relatively high contribution to kidney-absorbed dose from the lumbar region, uterus and ovary regions were incorporated into the male phantom to evaluate potential contribution to these regions from the lumbar CSF. Cross-radiation from the lumbar CSF to the uterus and ovaries was estimated at about $0.17 \%$ and $0.10 \%$, respectively. So, whereas lumbar CSF is the major contributing region to female reproductive organ-absorbed dose, its contribution is minimal. This study enrolled only male volunteers; no differences were observed in absorbed dose estimates in the testicles across the 2 groups.

\section{Biologic Implications}

Intrathecal administration results in a radioactivity distribution that differs from a typical intravenous injection. Radioactivity is highly localized and persistent in CSF regions. Thus, tissue-absorbed dose estimates in spinal CSF regions are high, particularly in the lumbar region. However, because tissuespecific ICRP weighting factors are not available for these regions, their contribution to effective dose is lumped into the remainder body compartment. Further, because of their small volume, the high local absorbed dose is diluted when considered alongside the volume of the entire body. Because relative absorbed dose to most, if not all, organs for which ICRP weighting factors exist is low, the resulting cumulative effective dose estimate is lower $(\sim 3 \mu \mathrm{Sv} / \mathrm{MBq})$ than for reported values from intravenous administration $(\sim 9 \mu \mathrm{Sv} / \mathrm{MBq}$ (29),).

The current experimentally derived dosimetry after intrathecal administration indicates an absorbed dose per unit injected activity of up to $0.8 \mathrm{mGy} / \mathrm{MBq}$ in the lumbar spine $(5-\mathrm{mL}$ injection, $n=3$ subjects). In this study, the administered activity of ${ }^{99 \mathrm{~m}} \mathrm{Tc}$ was about $185 \mathrm{MBq}$, resulting in a total radiation dose of about $140 \mathrm{mGy}(0.14 \mathrm{~Gy})$. This value is substantially below the threshold dose for radiation-induced myelopathy of $45 \mathrm{~Gy}$ and also below the lowest reported dose at which the L'hermitte syndrome has occurred. The lower thoracic, upper thoracic, and cervical spine regions receive lower dose than the lumbar region, although on the same order of magnitude. The brain CSF regions, particularly cisterns near the base of the brain and their adjacent structures, receive approximately an order of magnitude lower dose than the spine. Preliminary work evaluating injection volume

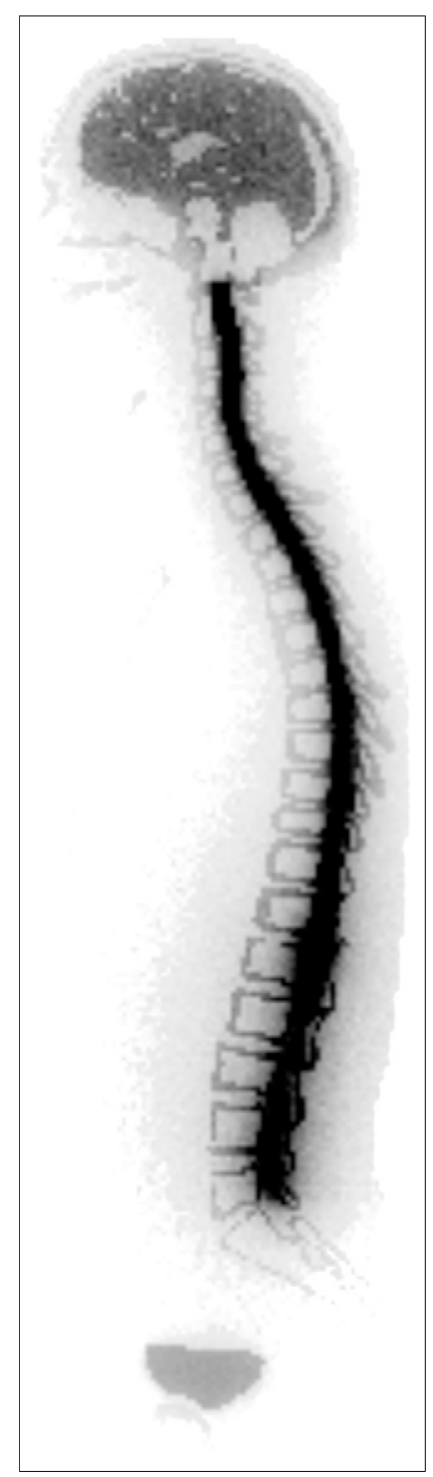

FIGURE 4. Absorbed dose per unit injected activity for average distribution of $5-\mathrm{mL}$ injected dose volume (maximum, $0.05 \mathrm{mGy} / \mathrm{MBq}$ ). also indicates that the dose may be decreased to the lumbar $(>50 \%)$ and thoracic regions $(\sim 25 \%)$ through the use of a larger injection volume $(\sim 0.3 \mathrm{mGy} / \mathrm{MBq}$ in lumbar region, 15-mL injection, $n=3$ subjects).

The most appropriate region for use in determining injected activity limits in intrathecal administration is a topic for further discussion. In this work, separate vertebral CSF subregions were evaluated. Alternately, given CSF movement and the observed administered volume effects, the average absorbed dose over the entire spine region may be a suitable region when considering dose-limiting organ. Given the estimated absorbed dose values observed here, particularly for small administered volume, that decision could be of relevance during study planning in terms of identifying whether a study is well suited to run under a Radioactive Drug Research Committee approval or should go to the Food and Drug Administration for approval. This evaluation may also be of greater importance for radionuclides that result in higher absorbed dose than ${ }^{99 m}$ Tc. A caveat is that subjects who have received radiation therapy to the spinal cord specifically, or to an area close to the spinal 
TABLE 3

Absorbed Dose Per Unit Injected Activity for Regions of Interest ( $\mu \mathrm{Gy} / \mathrm{MBq})$

\begin{tabular}{|lcc}
\hline \multicolumn{1}{c}{ Region } & \multicolumn{1}{c}{$5 \mathrm{~mL}$} & $15 \mathrm{~mL}$ \\
\hline Brain & $35.55 \pm 15.9$ & $50.19 \pm 4.29$ \\
\hline Right lung & $3.64 \pm 0.28$ & $2.84 \pm 0.33^{\star}$ \\
\hline Left lung & $3.49 \pm 0.26$ & $2.72 \pm 0.31^{\star}$ \\
\hline Liver & $3.28 \pm 0.34$ & $2.15 \pm 0.12^{\star}$ \\
\hline Kidneys & $13.70 \pm 1.29$ & $9.47 \pm 0.45^{\star}$ \\
\hline Stomach & $3.34 \pm 0.35$ & $2.23 \pm 0.12^{\star}$ \\
\hline Heart & $2.10 \pm 0.10$ & $1.54 \pm 0.12^{\star}$ \\
\hline Bladder & $5.96 \pm 2.01$ & $8.66 \pm 4.92$ \\
\hline Cervical CSF & $150.81 \pm 67.9$ & $178.04 \pm 30.55$ \\
\hline Thoracic CSF (upper) & $263.79 \pm 63.1$ & $207.26 \pm 62.17$ \\
\hline Thoracic CSF (lower) & $347.09 \pm 25.7$ & $268.99 \pm 17.29^{*}$ \\
\hline Lumbar CSF & $831.16 \pm 200.6$ & $373.66 \pm 69.65^{\star}$ \\
\hline Brain ventricle CSF & $27.00 \pm 12.04$ & $38.05 \pm 3.24$ \\
\hline Effective dose & $3.15 \pm 0.13$ & $2.79 \pm 0.10^{\star}$ \\
\hline
\end{tabular}

*Statistically significant differences (IS $t$ test, equal variance) observed between 5- and 15-mL groups.

Data are mean \pm SD.

cord, should be excluded on the basis that the additional radiation dose may result in a total dose greater than the threshold for the induction of either radiation myelopathy or the L'hermitte syndrome. Similarly, it would be prudent to exclude subjects who are receiving chemotherapy close to the time of the study.

In this study, absorbed dose maps have been generated for 9 source regions of interest for ${ }^{99 \mathrm{~m}} \mathrm{Tc}$. This methodology could be easily extended to additional source regions and radionuclides as dictated by study need. These additional regions and radionuclides will be of interest as the intrathecal route is evaluated for drug delivery and imaging applications. Additionally, the current spinal CSF source regions (lumbar, lower and upper thoracic, and cervical) could be further subdivided. Because of the small size of structures in this system relative to typical imaging resolution, some consideration would be required to incorporate, for example, the spinal cord or additional subregions of the leptomeningeal space separately from CSF.

\section{CONCLUSION}

We have extended the VIDA dosimetry framework and modified the human XCAT phantom to support radiation dose estimates from intrathecal administration. Simulation results were consistent with OLINDA results within $6 \%$ for shared regions. Application of this methodology to clinical data revealed a radiation dose effect dependent on volume of injected dose, with higher radiation dose observed at $5 \mathrm{~mL}$ than higher dose volumes, although additional studies may be required to corroborate these findings. Generally, radiation dose estimates are high in regions of the CSF and spinal cord because of the concentrated distribution of radionuclide in a confined volume that is distributed slowly along the CSF. Additionally, because these small regions do not have specific ICRP weighting factors, their contribution to effective dose is lumped into the remainder of the body and resulting effective dose estimates are potentially underestimated. This discrepancy should be considered when estimating dose in intrathecal injection.

\section{DISCLOSURE}

No potential conflict of interest relevant to this article was reported.

\section{ACKNOWLEDGMENTS}

We acknowledge Dr. Paul Segars for discussions and support around XCAT phantom modifications and Brendan Cook for his assistance in manuscript preparation.

\section{REFERENCES}

1. Calias P, Banks WA, Begley D, Scarpa M, Dickson P. Intrathecal delivery of protein therapeutics to the brain: a critical reassessment. Pharmacol Ther. 2014;144: 114-122.

2. Henry-Feugeas MC, Idy-peretti I, Baledent O, et al. Origin of subarachnoid cerebrospinal fluid pulsations: a phase-contrast MR analysis. Magn Reson Imaging. 2000;18:387-395.

3. Wåhlin A, Ambarki K, Hauksson J, Birgander R, Malm J, Eklund A. Phase contrast MRI quantification of pulsatile volumes of brain arteries, veins, and cerebrospinal fluids compartments: repeatability and physiological interactions. J Magn Reson Imaging. 2012;35:1055-1062.

4. Dreha-Kulaczewski S, Joseph AA, Merboldt KD, Ludwig HC, Gärtner J, Frahm J. Inspiration is the major regulator of human CSF flow. J Neurosci. 2015;35:2485-2491.

5. Iliff JJ, Wang M, Zeppenfeld DM, et al. Cerebral arterial pulsation drives paravascular CSF-interstitial fluid exchange in the murine brain. $J$ Neurosci. 2013;33: 18190-18199.

6. Sakka L, Coll G, Chazal J. Anatomy and physiology of cerebrospinal fluid. Eur Ann Otorhinolaryngol Head Neck Dis. 2011;128:309-316.

7. Wolf DA, Hesterman JY, Sullivan JM, et al. Dynamic dual-isotope molecular imaging elucidates principles for optimizing intrathecal drug delivery. JCI Insight. 2016;1:e85311.

8. Brenner DJ, Hall EJ. Computed tomography: an increasing source of radiation exposure. N Engl J Med. 2007;357:2277-2284.

9. Stabin MG, Sparks RB, Crowe E. OLINDA/EXM: the second-generation personal computer software for internal dose assessment in nuclear medicine. $\mathrm{J} \mathrm{Nucl}$ Med. 2005;46:1023-1027.

10. Stabin MG, Siegel JA. Physical models and dose factors for use in internal dose assessment. Health Phys. 2003;85:294-310.

11. Stabin MG, Flux G. Internal dosimetry as a tool for radiation protection of the patient in nuclear medicine. Biomed Imaging Interv J. 2007;3:1-11.

12. Tagesson M, Ljungberg M, Strand SE. A Monte-Carlo program converting activity distributions to absorbed dose distributions in a radionuclide treatment planning system. Acta Oncol. 1996;35:367-372.

13. Kolbert KS, Sgouros G, Scott AM, et al. Implementation and evaluation of patient-specific three-dimensional internal dosimetry. J Nucl Med. 1997;38: 301-308.

14. Prideaux AR, Song H, Hobbs RF, et al. Three-dimensional radiobiologic dosimetry: application of radiobiologic modeling to patient-specific 3dimensional imaging-based internal dosimetry. J Nucl Med. 2007;48:10081016.

15. Sgouros G, Frey E, Wahl R, He B, Prideaux A, Hobbs R. Three-dimensional imaging-based radiobiological dosimetry. Semin Nucl Med. 2008;38:321334.

16. Guy MJ, Flux GD, Papavasileiou P, Flower MA, Ott RJ. RMDP: a dedicated package for ${ }^{131}$ I SPECT quantification, registration and patient-specific dosimetry. Cancer Biother Radiopharm. 2003;18:61-69.

17. Chiavassa S, Bardiès M, Guiraud-vitaux F, et al. OEDIPE: a personalized dosimetric tool associating voxel-based models with MCNPX. Cancer Biother Radiopharm. 2005;20:325-332. 
18. Wilderman SJ, Dewaraja YK. Method for fast CT/SPECT-based 3D Monte Carlo absorbed dose computations in internal emitter therapy. IEEE Trans Nucl Sci. 2007;54:146-151.

19. Marcatili S, Pettinato C, Daniels S, et al. Development and validation of RAYDOSE: a Geant4-based application for molecular radiotherapy. Phys Med Biol. 2013;58:2491-2508.

20. Kost SD, Dewaraja YK, Abramson RG, Stabin MG. VIDA: a voxel-based dosimetry method for targeted radionuclide therapy using Geant4. Cancer Biother Radiopharm. 2015;30:16-26.

21. Sjögreen K, Ljungberg M, Wingårdh K, Minarik D, Strand SE. The LundADose method for planar image activity quantification and absorbed-dose assessment in radionuclide therapy. Cancer Biother Radiopharm. 2005;20:92-97.

22. Segars WP, Sturgeon G, Mendonca S, Grimes J, Tsui BM. 4D XCAT phantom for multimodality imaging research. Med Phys. 2010;37:4902-4915.

23. The International Commission on Radiological Protection (ICRP). The 2007 recommendations of the International Commission on Radiological Protection. ICRP publication 103. Ann ICRP. 2007;37:1-332.
24. White DR, Griffith RV, Wilson IJ. Photon, Electron, Proton, and Neutron Interaction Data for Body Tissues. ICRU 46. Bethesda, MD: International Commission on Radiation Units and Measurements; 1992.

25. Brownell GL, Ellett WH, Reddy AR. Absorbed fractions for photon dosimetry. J Nucl Med. 1968;suppl:29-39.

26. Ellett WH, Humes RM. Absorbed fractions for small volumes containing photon-emitting radioactivity. J Nucl Med. 1971;suppl 5:25-32.

27. Cristy M, Eckerman KF. Specific Absorbed Fractions of Energy at Various Ages from Internal Photon Sources. V5.ORNUTM/8381 A/5. Oak Ridge, TN: Oak Ridge National Laboratory; 1987.

28. Wolf D, Sullivan J, Mazur C, et al. The effect of bolus volume and mechanical forces on the biodistribution of ASOs following lumbar intrathecal administration in cynomolgus monkeys. Neurology. 2016;86(suppl): S38.007.

29. Stabin M, Taylor A, Eshima D, Wooter W. Radiation dosimetry for technetium-99m-MAG3, technetium-99m-DTPA, and iodine-131-OIH based on human biodistribution studies. J Nucl Med. 1992;33:33-40. 